\title{
METALES DE VALOR AÑADIDO EN LOS RÍOS SALADO UBICADOS EN LAS CUENCAS SAMA Y LOCUMBA (REGIÓN DE TACNA - PERÚ) Y SU CORRELACIÓN CON ARSÉNICO Y BORO
}

\author{
Edgardo Avendaño Cáceres ${ }^{* a}$, Margarita Briceño Toledo ${ }^{\mathrm{b}}$, Lorena Cornejo Ponce ${ }^{\mathrm{c}}$, \\ Dante Morales Cabrerad, Neil I. Ward ${ }^{*}$
}

\begin{abstract}
RESUMEN
Los objetivos de la presente investigación fueron identificar y cuantificar metales con valor añadido potencialmente comercializables de ser recuperados o extraídos en dos ríos denominados Salado; uno ubicado en la cuenca hidrográfica de Locumba (Salado-Locumba) y el otro en la cuenca hidrográfica de Sama (Salado-Sama). Además, realizar un análisis de correlación de los contenidos de arsénico (As) y boro (B) presentes en 14 puntos de muestreo ubicados en diferentes fuentes de agua a lo largo de las dos cuencas. Se analizaron 49 elementos utilizando ICP-MS, en muestras de agua para determinar metales totales y disueltos. En los análisis de correlación, asociación y reducción de variables se aplicaron métodos estadísticos robustos. Los metales de valor añadido identificados fueron: $\mathrm{Li}, \mathrm{Rb}$, Cs y Ge en concentraciones de 1,818 $\pm 0,415 \mathrm{mg} / \mathrm{L}, 0,248 \pm 0,048 \mathrm{mg} / \mathrm{L}, 0,361 \pm 0,104 \mathrm{mg} / \mathrm{L}$ y $0,005 \pm 0,002 \mathrm{mg} / \mathrm{L}$ en el río Salado-Locumba y 1,950 $\pm 0,544 \mathrm{mg} / \mathrm{L}, 0,343 \pm 0,090 \mathrm{mg} / \mathrm{L}$, $0,401 \pm 0,096 \mathrm{mg} / \mathrm{L}$ y $0,005 \pm 0,002 \mathrm{mg} / \mathrm{L}$ en el río Salado-Sama, respectivamente. Se determinó que la correlación entre las concentraciones de los elementos químicos de valor añadido $\mathrm{Li}$, $\mathrm{Rb}$, Cs y los elementos As y B presentes en 14 puntos de muestreo ubicados en diferentes fuentes de agua a lo largo de las dos cuencas, son estadísticamente significativos, positivos y altos $(r>0,7$ y $p<0,01)$.
\end{abstract}

Palabras clave: metales de valor añadido, litio, rubidio, cesio y germanio.

\footnotetext{
a Departamento de Química e Ingeniería Química, Facultad de Ingeniería, Universidad Nacional Jorge Basadre Grohman, Av. Miraflores s/n, Tacna, Perú. 23001.

*eavendanoc@unjbg.edu.pe

b Facultad de Ciencias de la Salud, Universidad Arturo Prat, Iquique, Chile.

c Departamento de Ingeniería Mecánica, Facultad de Ingeniería, Universidad de Tarapacá, Arica, Chile.

d Facultad de Ingeniería, Universidad Nacional Jorge Basadre Grohman,

e Department of Chemistry, FEPS, University of Surrey, Guildford, Surrey, United Kingdom.
} 


\title{
ADDED-VALUE METALS IN THE SALADO RIVERS LOCATED IN THE SAMA AND LOCUMBA BASINS (TACNA REGION - PERU) AND THEIR CORRELATION WITH ARSENIC AND BORON
}

\begin{abstract}
The objectives of this research were to identify and quantify potentially marketable added value metals, if recovered or extracted, in two rivers called Salado; one located in the Locumba basin (Salado-Locumba) and the other in the Sama basin (Salado-Sama). In addition, perform a correlation analysis of the arsenic (As) and boron (B) contents present in 14 sampling points located in different water sources throughout the two basins. Total and dissolved metals (49) in samples were analyzed using ICP-MS. Robust statistical methods were applied in the correlation, association and reduction analysis of variables. The identified value-added metals were: $\mathrm{Li}, \mathrm{Rb}, \mathrm{Cs}$ and $\mathrm{Ge}$ in concentrations of $1,818 \pm 0,415 \mathrm{mg} / \mathrm{L}, 0,248 \pm 0,048 \mathrm{mg} / \mathrm{L}$, $0,361 \pm 0,104 \mathrm{mg} / \mathrm{L}$ and $0,005 \pm 0,002 \mathrm{mg} / \mathrm{L}$ in the Salado-Locumba river, and 1,950 $\pm 0,544$ $\mathrm{mg} / \mathrm{L}, 0,343 \pm 0,090 \mathrm{mg} / \mathrm{L}, 0,401 \pm 0,096 \mathrm{mg} / \mathrm{L}$ and $0,005 \pm 0,002 \mathrm{mg} / \mathrm{L}$ in the Salado-Sama river, respectively. It was determined that the correlation between the concentrations of the added value chemical elements $\mathrm{Li}, \mathrm{Rb}, \mathrm{Cs}$ and the elements As and B present in 14 sampling points located in different water sources along the two basins the two basins, are statistically significant, positive and high $(\mathrm{r}>0,7$ and $\mathrm{p}<0,01)$.
\end{abstract}

Keywords: value added metals, lithium, rubidium, cesium and germanium

\section{INTRODUCCIÓN}

La principal forma de ingreso del arsénico, al cuerpo humano, es a través del agua o alimentos contaminados con dicho elemento ${ }^{(1)}$, por lo que una ingesta excesiva puede llegar a producir carcinogénesis. Algunos autores ${ }^{(2)}$ mencionan que las concentraciones elevadas de arsénico encontradas en las fuentes destinadas para consumo humano en varios países a nivel mundial han causado un impacto importante en la salud pública; entre los países de América Latina con esta problemática tenemos a Argentina, Bolivia, Chile, México y Perú. En el mapa de distribución de acuíferos y cuerpos de agua superficiales con altos contenidos de arsénico disuelto en América Latina ${ }^{(2)}$, se ubican las zonas del Perú. La región de Tacna es una de las zonas descritas con esta problemática especificando, entre otras, el valle de Locumba, Sama Inclán y Sama Las Yaras (zonas que se encuentran ubicadas en las cuencas hidrográficas de Locumba y Sama localizadas en la zona rural de Tacna).

En la región de Tacna se han identificado dos tributarios de nombre Salado; uno ubicado en la cuenca hidrográfica Locumba (río Salado-Locumba) y el otro en la cuenca hidrográfica Sama (río Salado-Sama). En los resultados de los análisis fisicoquímicos ejecutados en época de estiaje por el monitoreo participativo de calidad de agua superficial en los años 2016, 2019 y 2020 en las cuencas hidrográficas de Sama y Locumba; proporcionados por la Autoridad 
Nacional del Agua (ANA) a través de la Administración Local del Agua (ALA) CaplinaLocumba, en ambos ríos (Salado-Locumba y Salado-Sama) se ha identificado y cuantificado altos contenidos de $\mathrm{As}, \mathrm{B}$ y $\mathrm{Li}$, entre otros elementos, contribuyendo a incrementar la concentración de estos elementos en diferentes tributarios. Estos dos ríos se conforman por el tránsito de la precipitación y la actividad hidrotermal de la zona que ocurre en la parte alta de las dos cuencas. Normalmente las aguas de origen geotérmico en su composición muestran entre otros elementos adicionales a los antes mencionados, metales de valor añadido. Son elementos que al no encontrarse comúnmente en la corteza terrestre requieren métodos no convencionales para su extracción y presentan un alto valor en el mercado internacional. Adicionalmente, existe información de estudios, investigaciones, procesos y operaciones unitarias patentadas acerca de la recuperación de varios de estos elementos en agua de mar, agua de rechazo o salmueras de diversos procesos industriales ${ }^{(3)}$. Entre los más importantes para la presente investigación se encuentran el $\mathrm{Rb}, \mathrm{Cs}$, Ge y $\mathrm{Li}$, de estos metales la ANA, durante sus monitoreos participativos anuales de calidad de agua, solamente reporta el monitoreo del Li en las fuentes superficiales de las cuencas hidrográficas de Locumba y Sama.

La presente investigación tiene como objetivo identificar y cuantificar los niveles de metales de valor añadido en los ríos Salado-Locumba y Salado-Sama, como potenciales metales comercializables, después de ser recuperados o extraídos. Además, realizar un análisis de correlación entre los contenidos de los metales de valor añadido y los elementos As y B presentes en los 14 puntos de muestreo ubicados en diferentes fuentes de agua a lo largo de las dos cuencas.

\section{PARTE EXPERIMENTAL}

\section{1 Área de estudio}

La figura 1 muestra las cuencas hidrográficas de Locumba y Sama con los colores verde y rosado respectivamente. La cuenca hidrográfica del río Sama tiene dos zonas diferenciadas: la cuenca alta, cuyos ríos principales son el Salado y el río Tala que es el resultado de la unión de los ríos Ticalaco, Pistala y el río de Estique-Tarucachi-Talabaya. La cuenca media y baja, está formada por el río Sama resultado de la unión de los ríos Salado y Tala. Esta cuenca tiene tres trasvases: Barroso hacia la cuenca Caplina, túnel Kovire al río Maure y finalmente Dique Cano-Salado hacia la cuenca Locumba. Es importante mencionar que el trasvase de Kovire beneficia en menor proporción de agua a la cuenca Sama y en una mayor proporción a la cuenca Locumba, siendo esta agua trasvasada a la laguna de Aricota ubicada en esta última cuenca ${ }^{(4)}$. 


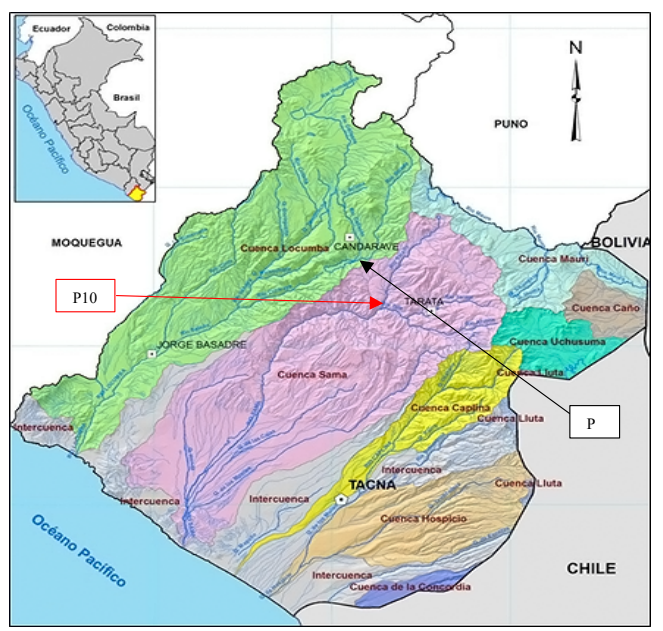

Figura 1. Cuencas hidrográficas de la región Tacna. Las cuencas hidrográficas Locumba y Sama están representadas por las áreas de color verde y rosado respectivamente. P4 (río Salado-Locumba antes de la unión con el río Callazas) y P10 (río Salado-Sama antes de la unión con el río Tala). Fuente: INGEMMET ${ }^{(5)}$

El río Salado-Sama, es un río que se conforma por el tránsito de la precipitación y la actividad hidrotermal que se lleva a cabo en la subcuenca denominada Salado la misma que involucra al aporte de varias microcuencas como Ichicollo, Cano, Canocucho, Yabroco, Challahuaya entre otros. Este río se caracteriza por presentar actividad hidrotermal (aguas calientes con alto contenido de arsénico, boro y otros minerales disueltos), así mismo es una cuenca que presenta aportes hídricos de la derivación de la cuenca del río Maure específicamente del río Ancoaque, las mismas que se realizan mediante el túnel Kovire; recursos hídricos que en el dique Cano una parte son derivadas hacia la cuenca del río Sama por el río salado y la otra a la cuenca Locumba.

La cuenca hidrográfica del río Locumba tiene tres zonas diferenciadas: cuenca alta, inicia desde la laguna Suches y llega hasta la laguna, siendo conformada por los ríos Callazas, Matazas, Salado y Tacalaya. La cuenca Media, está conformada por los ríos Curibaya, Ilabaya y Cinto, hasta los límites de la población de Camiara; toda esta zona sería el valle de Locumba. La cuenca Baja, es la zona de Ite, conformada por la desembocadura en los humedales de la bahía del mismo nombre. Esta cuenca hidrográfica tiene los trasvases desde la cuenca alta del río Maure hacia la laguna de Aricota mediante el túnel Ichicollo. También tiene trasvases de recurso subterráneo y superficial de las lagunas Suches, Vizcachas y otros acuíferos asociados hacia las minas de Toquepala (subcuenca distinta) y Cuajone. Finalmente, la cuenca baja tiene el trasvase hacia la ciudad de Ilo (Moquegua) $)^{(4)}$. 
El río Salado ubicado en la cuenca hidrográfica de Locumba confluye con el río Callazas para desembocar en la laguna de Aricota. Es resultado de la mezcla de las aguas que se transfieren de la cuenca Sama, a través del túnel Ichicollo a la cuenca hidrográfica de Locumba; estas son descargadas al río Jaruma, para luego en su transcurso mezclarse con el río Calientes para formar el río Salado. Las características fisicoquímicas del río Jaruma en cuanto al arsénico y otros elementos son debido a la naturaleza geológica y surgencias geotermales propias de la cuenca Calientes-Locumba ${ }^{(6)}$.

\subsection{Selección de puntos de muestreo en los ríos Salado de las cuencas Sama y Locumba}

Para determinar los puntos de muestreo, se realizó una búsqueda sistemática de información de calidad fisicoquímica del agua correspondiente a los ríos Salado-Locumba y Salado-Sama. La información fue analizada de los resultados del monitoreo participativo de calidad de agua superficial en la cuenca Sama y Locumba durante los años 2016 - 2020, en temporada de estiaje (meses de mediados de junio a mediados de diciembre), proporcionados por la ANAALA-Caplina-Locumba. Los puntos de muestreo Salado-Locumba y Salado-Sama están identificados en la red de monitoreo de la ANA-ALA-Caplina-Locumba como: en la cuenca hidrográfica Sama 13158RSala2 (río salado antes de la confluencia con el río Tala, Localidad de Chucatamani, distrito Héroes Albarracín, provincia de Tarata, coordenadas UTM 8067623 Norte, 375741 Este) y en la cuenca hidrográfica Locumba 1316RSala1 (río salado antes de la confluencia con el río Callazas, Localidad y distrito de Quilahuani, provincia de Candarave, coordenadas UTM 369860 Este y 8083353 Norte).

Adicionalmente, se estableció puntos de muestreo complementarios en diferentes fuentes de agua a lo largo de las dos cuencas teniendo como referencia los puntos seleccionados por la ANA. Lo anterior, con el fin de tener un valor aproximado de la distribución de los metales de valor añadido a lo largo de las dos cuencas y hacer un análisis correlación de los mismos con los elementos arsénico y boro. Estos muestreos fueron realizados una sola vez y en las muestras de agua se analizaron metales totales. La ubicación de los muestreos se observa en la figura 2. 


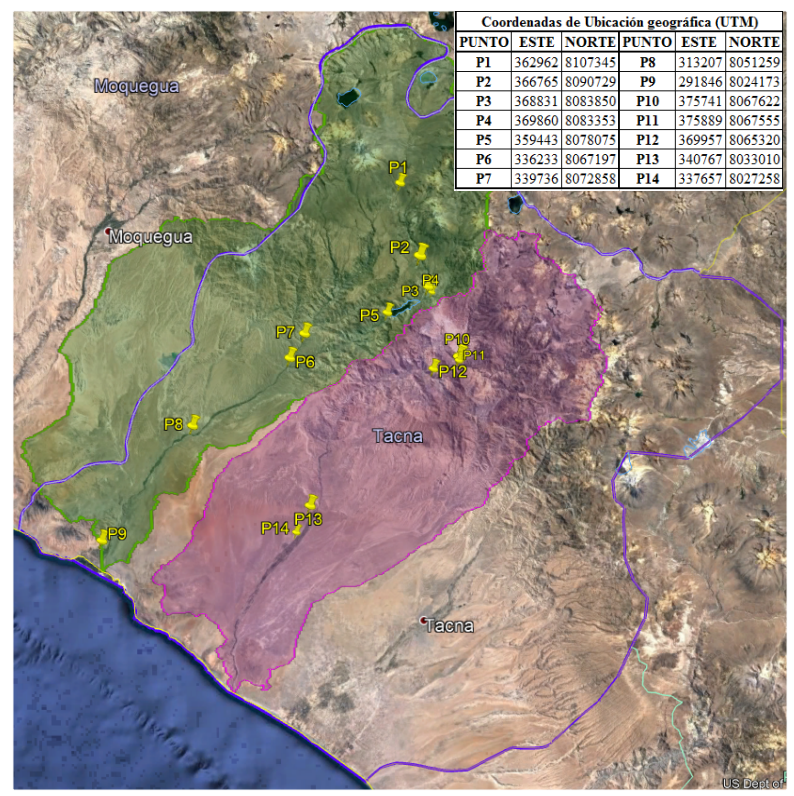

Figura 2. Puntos de muestreo (P) en diferentes fuentes de agua a lo largo de las cuencas hidrográficas Locumba (P1 a P9) y Sama (P10 a P14) en la región de Tacna. P4 y P10 corresponden a los ríos Salado-Locumba y Salado-Sama respectivamente. Fuente: Imagen Satelital extraída de Google Earth Pro.

\subsection{Muestreo y análisis de laboratorio:}

Se consideraron 14 puntos de muestreo a lo largo de las cuencas hidrográficas de Locumba y Sama distribuidos en 09 y 05, respectivamente. Para los muestreos se utilizó el protocolo Nacional de Monitoreo de la Calidad de los Recursos Hídricos Superficiales de la ANA ${ }^{(7)}$. Las muestras de agua fueron enviadas para su análisis al laboratorio acreditado SGS del Perú S.A.C., analizándose 49 elementos químicos utilizando para la determinación la técnica ICPMS (modelo NEXION 300D marca Perking Elmer). Los parámetros de campo considerados fueron $\mathrm{pH}$, conductividad eléctrica y temperatura, para lo cual se utilizó un multiparámetro (marca HANNA modelo HI98129), finalmente, para la ubicación de los puntos de muestreo se utilizó un equipo de posicionamiento global (GPS, marca Garmin modelo Montana 680). El método de ensayo utilizado para el análisis de metales totales y disueltos fue el EPA 200.8, Rev 5.4:1994. Para el análisis de metales solubles las muestras fueron filtradas inmediatamente después de su muestreo utilizando filtro de jeringa no estéril de Nylon de 25 $\mathrm{mm}, 0,45 \mu \mathrm{m}$, marca Pall. El preservante utilizado para las muestras destinadas al análisis de metales totales y solubles fue $\mathrm{HNO}_{3}$ al $1 \%(\mathrm{v} / \mathrm{v})$, las muestras fueron mantenidas refrigeradas a $4^{\circ} \mathrm{C}$ en un cooler y enviadas para su análisis al laboratorio acreditado de SGS del Perú S.A.C. 
Para la identificación y cuantificación de los metales de valor añadido en los ríos Salado de las cuencas hidrográficas Locumba y Sama se realizó dos tipos de análisis en las mismas muestras: un análisis de metales totales y otro análisis de metales solubles (las muestras se filtraron in situ utilizando filtros de jeringa de nylon de $0,45 \mu \mathrm{m})$. Se realizó 03 muestreos de metales totales por cada río Salado con diferencias aproximadamente de un mes (septiembrediciembre del 2020), en dos de las tres campañas de muestreo se realizó los análisis de metales totales y solubles (meses de septiembre y diciembre). Para la determinación del porcentaje de la fracción insoluble de los metales; en cada una de las dos muestras se calculó la diferencia entre los valores que superaron el límite de cuantificación (LC) de los metales totales y los metales solubles; los dos resultados se promediaron y permitieron calcular el porcentaje de la fracción insoluble de los metales totales en los dos ríos Salado.

\subsection{Procesamiento de datos}

Para el análisis de correlación se descartó los elementos que mostraron más del $40 \%$ de sus datos con valores iguales al límite de cuantificación (LC). Posteriormente, se aplicaron las pruebas de normalidad de Shapiro-Wilk. Los elementos cuyos valores no tuvieron una distribución normal se les aplicó la transformación de Box y Cox y fueron codificados anteponiendo la letra T, finalmente, al análisis conjunto de datos se aplicó la correlación de Pearson. En el caso de los análisis multivariados de agrupación y clasificación de los puntos de muestreo y sus elementos (individuos y variables) se utilizó el análisis de conglomerado jerárquico (HCA) con el comando "hcut" aplicando el método de Ward.D2 y la métrica de Manhattan para cuantificación de la similitud, permitiendo construir el dendograma. Como método de reducción de variables se aplicó el método de análisis de componentes principales robusto (ROBPCA - ROBust) utilizando el comando "PcaHubert". Para el cálculo de la correlación robusta se utilizó el comando "CovOgk". Los comandos hcut, PcaHubert y CovOgK se encuentran implementados en el software R.

\section{RESULTADOS Y DISCUSIÓN}

\subsection{Identificación y cuantificación de metales de valor añadido en los ríos Salado- Locumba y Salado-Sama}

Se puede observar en las tablas 1 y 2 las características fisicoquímicas de las 14 fuentes diferentes de agua ubicadas a lo largo de las cuencas hidrográficas Locumba y Sama y los valores de los contenidos de los metales y metaloides que corresponden a metales totales. Los ríos Salado están identificados con código P4(Salado-Locumba) y P10(Salado-Sama) en ambos casos se muestra el promedio junto a su desviación estándar resultado de 03 campañas de muestreo $(n=3)$. Los metales de valor añadido motivo de estudio Li, Rb, Cs y Ge alcanzaron valores promedio en el río Salado-Locumba (P4) de 1,818 mg/L, 0,2484 mg/L, 0,3606 mg/L y $0,0047 \mathrm{mg} / \mathrm{L}$ respectivamente. En el río Salado-Sama (P10) alcanzarón valores promedio de $1,9501 \mathrm{mg} / \mathrm{L}, 0,3427 \mathrm{mg} / \mathrm{L}, 0,4014 \mathrm{mg} / \mathrm{L}$, y $0,0049 \mathrm{mg} / \mathrm{L}$ respectivamente. De los 14 puntos muestreados, los dos ríos Salado presentaron la mayor concentración de $\mathrm{Li}, \mathrm{Rb}, \mathrm{Cs}$ y Ge. La presencia de estos elementos es debido a que estos ríos al conformase por el tránsito de la precipitación y la actividad hidrotermal pasan en su trayecto por zonas volcánicas propias de 
la naturaleza geológica de las dos cuencas hidrográficas. Como también lo muestran otros estudios de aguas geotermales en diferentes países ${ }^{(8)(9)}$.

Tabla 1. Análisis fisicoquímico de las muestras de agua en la cuenca hidrográfica de Locumba.

\begin{tabular}{|c|c|c|c|c|c|c|c|c|c|}
\hline \multirow[b]{2}{*}{ Parámetros } & \multicolumn{9}{|c|}{ Puntos de muestreo y fechas de muestreo } \\
\hline & $\begin{array}{c}P 1(n=1) \\
19 / 10 / 2020\end{array}$ & $\begin{array}{c}\text { P2(n=1) } \\
17 / 12 / 2020\end{array}$ & $\begin{array}{c}\text { P3(n }=1) \\
09 / 12 / 2020\end{array}$ & $\begin{array}{c}\mathbf{P 4}(\mathrm{n}=3) \\
*\end{array}$ & $\begin{array}{c}\text { P5(n=1) } \\
09 / 12 / 2020\end{array}$ & $\begin{array}{c}\text { P6(n=1) } \\
20 / 10 / 2020\end{array}$ & $\begin{array}{c}P 7(n=1) \\
20 / 10 / 2020\end{array}$ & $\begin{array}{c}P 8(n=1) \\
31 / 10 / 2020\end{array}$ & $\begin{array}{c}P 9(n=1) \\
14 / 10 / 2020\end{array}$ \\
\hline $\mathrm{T},{ }^{\circ} \mathrm{C}$ & 16,8 & 15,5 & 18,6 & $18,5 \pm 2,2$ & 18,1 & 19,7 & 24,5 & 26,11 & 18,79 \\
\hline $\mathrm{pH}$ & 8,13 & 6,94 & 8,52 & $8,28 \pm 0,05$ & 8,57 & 8,18 & 8,47 & 8,43 & 8,3 \\
\hline $\mathrm{CE}, \mathrm{uS} / \mathrm{cm}$ & 2174 & 1055 & 1308 & $2760 \pm 163$ & 1784 & 1859 & 1417 & 2268 & 2274 \\
\hline $\mathrm{Al}, \mathrm{mg} / \mathrm{L}$ & 1,116 & $<0,003$ & 0,076 & $1,771 \pm 2,019$ & $<0,003$ & 0,068 & 0,044 & 0,336 & 1,096 \\
\hline As, $\mathrm{mg} / \mathrm{L}$ & 1,19581 & 0,53964 & 0,48219 & $1,52576 \pm 0,28293$ & 0,64663 & 0,71569 & 0,09031 & 0,60067 & 0,53810 \\
\hline $\mathrm{B}, \mathrm{mg} / \mathrm{L}$ & 3,979 & 0,027 & 5,576 & $11,149 \pm 1,454$ & 8,396 & 8,841 & 4,037 & 9,235 & 6,293 \\
\hline $\mathrm{Ba}, \mathrm{mg} / \mathrm{L}$ & 0,0187 & 0,0870 & 0,0226 & $0,0394 \pm 0,0064$ & 0,0426 & 0,0377 & 0,0404 & 0,0484 & 0,0370 \\
\hline $\mathrm{Ca}, \mathrm{mg} / \mathrm{L}$ & 36,950 & 62,996 & 69,85 & $103,61 \pm 2,62$ & 153,835 & 112,000 & 133,000 & 146,000 & 131,085 \\
\hline $\mathrm{Ce}, \mathrm{mg} / \mathrm{L}$ & 0,00051 & $<0,00024$ & 0,00012 & $0,00064 \pm 0,00077$ & $<0,00024$ & $<0,00024$ & $<0,00024$ & 0,00083 & 0,00182 \\
\hline $\mathrm{Co}, \mathrm{mg} / \mathrm{L}$ & 0,00043 & 0,00011 & 0,000015 & $0,00215 \pm 0,00223$ & 0,00438 & $<0,00003$ & 0,00014 & 0,00033 & 0,00057 \\
\hline $\mathrm{Cs}, \mathrm{mg} / \mathrm{L}$ & 0,1327 & 0,0019 & 0,0306 & $0,3606 \pm 0,1036$ & 0,1623 & 0,1710 & 0,0086 & 0,1236 & 0,0668 \\
\hline $\mathrm{Cu}, \mathrm{mg} / \mathrm{L}$ & 0,00191 & $<0,00009$ & 0,000045 & $<0,00009$ & $<0,00009$ & 0,00234 & 0,00419 & 0,00233 & 0,00338 \\
\hline $\mathrm{Fe}, \mathrm{mg} / \mathrm{L}$ & 1,6316 & $<0,0013$ & 0,0919 & $1,259 \pm 1,02$ & 0,0037 & 0,0575 & 0,0541 & 0,3223 & 1,1078 \\
\hline $\mathrm{Ga}, \mathrm{mg} / \mathrm{L}$ & $<0,00012$ & $<0,00012$ & $<0,00012$ & $<0,00012$ & 0,00017 & $<0,00012$ & $<0,00012$ & 0,00017 & 0,00028 \\
\hline $\mathrm{Ge}, \mathrm{mg} / \mathrm{L}$ & 0,0040 & $<0,0006$ & $<0,0006$ & $0,0047 \pm 0,0017$ & 0,0009 & 0,0013 & $<0,0006$ & $<0,0006$ & $<0,0006$ \\
\hline $\mathrm{Hf}, \mathrm{mg} / \mathrm{L}$ & 0,00020 & $<0,00015$ & $<0,00015$ & $0,00041 \pm 0,00045$ & 0,00839 & 0,00041 & 0,00040 & $<0,00015$ & $<0,00015$ \\
\hline $\mathrm{K}, \mathrm{mg} / \mathrm{L}$ & 23,80 & 17,15 & 18,89 & $51,46 \pm 1,43$ & 46,46 & 32,75 & 14,55 & 34,45 & 21,41 \\
\hline $\mathrm{Li}, \mathrm{mg} / \mathrm{L}$ & 0,6172 & 0,2503 & 0,3678 & $1,818 \pm 0,415$ & 1,3182 & 1,1370 & 0,2694 & 1,1070 & 0,7299 \\
\hline $\mathrm{Mg}, \mathrm{mg} / \mathrm{L}$ & 13,800 & 19,170 & 29,298 & $36,669 \pm 3,19$ & 27,390 & 33,600 & 33,550 & 39,900 & 36,255 \\
\hline $\mathrm{Mn}, \mathrm{mg} / \mathrm{L}$ & 0,32742 & $<0,00010$ & 0,00881 & $0,22456 \pm 0,06753$ & 0,00303 & 0,01369 & 0,03428 & 0,06500 & 0,14755 \\
\hline $\mathrm{Mo}, \mathrm{mg} / \mathrm{L}$ & 0,00516 & 0,00117 & 0,00332 & $0,00844 \pm 0,00245$ & 0,00913 & 0,00832 & 0,00508 & 0,00955 & 0,00640 \\
\hline $\mathrm{Na}, \mathrm{mg} / \mathrm{L}$ & 152,500 & 132,783 & 166,786 & $382,716 \pm 44,003$ & 181,742 & 251,000 & 164,500 & 275,000 & 271,773 \\
\hline $\mathrm{Ni}, \mathrm{mg} / \mathrm{L}$ & 0,0007 & $<0,0006$ & $<0,0006$ & $0,003 \pm 0,0032$ & $<0,0006$ & $<0,0006$ & $<0,0006$ & 0,0008 & $<0,0006$ \\
\hline $\mathrm{Rb}, \mathrm{mg} / \mathrm{L}$ & 0,1142 & 0,0107 & 0,0449 & $0,2484 \pm 0,0477$ & 0,1521 & 0,1431 & 0,0333 & 0,1390 & 0,0891 \\
\hline $\mathrm{Sb}, \mathrm{mg} / \mathrm{L}$ & 0,01560 & 0,00158 & 0,0027 & $0,06221 \pm 0,01375$ & 0,03017 & 0,02000 & $<0,00013$ & 0,01570 & 0,00728 \\
\hline $\mathrm{Se}, \mathrm{mg} / \mathrm{L}$ & $<0,0013$ & $<0,0013$ & $<0,0013$ & $0,0022 \pm 0,0014$ & $<0,0013$ & 0,0034 & 0,0021 & 0,0041 & $<0,0013$ \\
\hline $\mathrm{Si}, \mathrm{mg} / \mathrm{L}$ & 67,80 & 36,86 & 36,06 & $61,16 \pm 16,17$ & 48,91 & 34,41 & 26,08 & 36,95 & 26,39 \\
\hline $\mathrm{SiO}_{2}, \mathrm{mg} / \mathrm{L}$ & 145,06 & 78,86 & 77,14 & $130,83 \pm 34,59$ & 104,63 & 73,61 & 55,79 & 79,05 & 56,45 \\
\hline $\mathrm{Sr}, \mathrm{mg} / \mathrm{L}$ & 0,2489 & 0,3699 & 0,4322 & $0,8411 \pm 0,0862$ & 0,8881 & 0,7995 & 0,9130 & 1,0468 & 0,7942 \\
\hline $\mathrm{Ti}, \mathrm{mg} / \mathrm{L}$ & 0,0103 & 0,0035 & 0,0041 & $0,0096 \pm 0,0041$ & 0,0042 & 0,0055 & 0,0038 & 0,0182 & 0,0381 \\
\hline $\mathrm{U}, \mathrm{mg} / \mathrm{L}$ & 0,000477 & 0,001668 & 0,001084 & $0,00025 \pm 0,000214$ & 0,001640 & 0,001856 & 0,004993 & 0,003132 & 0,003231 \\
\hline $\mathrm{V}, \mathrm{mg} / \mathrm{L}$ & 0,0075 & 0,0062 & 0,0086 & $0,006 \pm 0,0013$ & 0,0023 & 0,0052 & 0,0078 & 0,0092 & 0,0093 \\
\hline $\mathrm{W}, \mathrm{mg} / \mathrm{L}$ & 0,0071 & $<0,0006$ & $<0,0006$ & $0,015 \pm 0,0184$ & 0,0038 & 0,0112 & 0,0200 & 0,0007 & $<0,0006$ \\
\hline $\mathrm{Yb}, \mathrm{mg} / \mathrm{L}$ & 0,00010 & $<0,00006$ & $<0,00006$ & $<0,00006$ & $<0,00006$ & $<0,00006$ & $<0,00006$ & $<0,00006$ & $<0,00006$ \\
\hline $\mathrm{Zn}, \mathrm{mg} / \mathrm{L}$ & 0,0348 & $<0,0026$ & 0,0027 & $0,0199 \pm 0,0223$ & $<0,0026$ & 0,0298 & 0,0145 & 0,0032 & 0,0041 \\
\hline $\mathrm{Zr}, \mathrm{mg} / \mathrm{L}$ & $<0,00045$ & $<0,00045$ & $<0,00045$ & $<0,00045$ & 0,00393 & $<0,00045$ & $<0,00045$ & $<0,00045$ & 0,00068 \\
\hline
\end{tabular}

n: Número de muestreos realizados durante la investigación. La desviación estándar en P4 se determinó en base a 03 muestreos.

*: 26/09/2020,19/10/2020 y 09/12/2020 (fechas de muestreo) 
Tabla 2. Análisis fisicoquímico de las muestras de agua en la cuenca hidrográfica de Sama.

\begin{tabular}{|c|c|c|c|c|c|}
\hline \multirow[b]{2}{*}{ Parámetros } & \multicolumn{5}{|c|}{ Puntos de muestreo y fechas de muestreo } \\
\hline & $\underset{* *}{P 10}(\mathbf{n}=\mathbf{3})$ & $\begin{array}{c}\text { P11(n=1) } \\
20 / 10 / 2020\end{array}$ & $\begin{array}{c}\text { P12(n=1) } \\
09 / 12 / 2020\end{array}$ & $\begin{array}{c}P 13(n=1) \\
14 / 10 / 2020\end{array}$ & $\begin{array}{c}\text { P14(n=1) } \\
14 / 10 / 2020\end{array}$ \\
\hline $\mathrm{T},{ }^{\circ} \mathrm{C}$ & $21 \pm 1$ & 21 & 22,3 & 18,17 & 19,5 \\
\hline $\mathrm{pH}$ & $8,43 \pm 0,15$ & 8,25 & 8,43 & 8,33 & 8,2 \\
\hline $\mathrm{CE}, \mathrm{uS} / \mathrm{cm}$ & $2652 \pm 109$ & 1406 & 2073 & 2211 & 2505 \\
\hline $\mathrm{Al}, \mathrm{mg} / \mathrm{L}$ & $0,067 \pm 0,04$ & 0,496 & 0,912 & 0,286 & 0,265 \\
\hline As, mg/L & $1,2612 \pm 0,14663$ & 0,03796 & 0,45061 & 0,34334 & 0,34249 \\
\hline $\mathrm{B}, \mathrm{mg} / \mathrm{L}$ & $11,299 \pm 2,002$ & 1,428 & 8,703 & 5,171 & 5,192 \\
\hline $\mathrm{Ba}, \mathrm{mg} / \mathrm{L}$ & $0,0417 \pm 0,0085$ & 0,0295 & 0,0278 & 0,0284 & 0,0288 \\
\hline $\mathrm{Be}, \mathrm{mg} / \mathrm{L}$ & $<0,00006$ & 0,00003 & 0,00013 & $<0,00006$ & $<0,00006$ \\
\hline $\mathrm{Ca}, \mathrm{mg} / \mathrm{L}$ & $122,662 \pm 12,946$ & 245,5 & 174,487 & 184,992 & 186,966 \\
\hline $\mathrm{Ce}, \mathrm{mg} / \mathrm{L}$ & $<0,00024$ & 0,00146 & 0,00147 & 0,00041 & 0,00034 \\
\hline $\mathrm{Co}, \mathrm{mg} / \mathrm{L}$ & $0,00011 \pm 0,00008$ & 0,00598 & 0,00282 & 0,00071 & 0,00055 \\
\hline $\mathrm{Cs}, \mathrm{mg} / \mathrm{L}$ & $0,4014 \pm 0,0958$ & 0,0032 & 0,1798 & 0,1009 & 0,1025 \\
\hline $\mathrm{Cu}, \mathrm{mg} / \mathrm{L}$ & $<0,00009$ & 0,00513 & 0,00365 & $<0,00009$ & $<0.00009$ \\
\hline $\mathrm{Fe}, \mathrm{mg} / \mathrm{L}$ & $0,225 \pm 0,0242$ & 0,5512 & 1,0125 & 0,4607 & 0,4033 \\
\hline $\mathrm{Ga}, \mathrm{mg} / \mathrm{L}$ & $<0,00012$ & 0,00006 & 0,00021 & $<0,00012$ & $<0,00012$ \\
\hline $\mathrm{Ge}, \mathrm{mg} / \mathrm{L}$ & $0,0049 \pm 0,0016$ & $<0,0006$ & 0,0013 & $<0,0006$ & $<0,0006$ \\
\hline $\mathrm{Hf}, \mathrm{mg} / \mathrm{L}$ & $<0,00015$ & 0,00025 & $<0,00015$ & $<0,00015$ & $<0,00015$ \\
\hline $\mathrm{K}, \mathrm{mg} / \mathrm{L}$ & $57,02 \pm 4,45$ & 6 & 25,35 & 17,63 & 18,31 \\
\hline $\mathrm{Li}, \mathrm{mg} / \mathrm{L}$ & $1,9501 \pm 0,5445$ & 0,1383 & 0,7884 & 0,7275 & 0,7427 \\
\hline $\mathrm{Mg}, \mathrm{mg} / \mathrm{L}$ & $26,19 \pm 2,48$ & 57 & 40,578 & 36,033 & 36,999 \\
\hline $\mathrm{Mn}, \mathrm{mg} / \mathrm{L}$ & $0,3606 \pm 0,0502$ & 0,28665 & 0,28043 & 0,12526 & 0,09386 \\
\hline Mo, mg/L & $0,00322 \pm 0,00132$ & 0,00264 & 0,00144 & 0,00321 & 0,00324 \\
\hline $\mathrm{Na}, \mathrm{mg} / \mathrm{L}$ & $359,895 \pm 55,107$ & 76,5 & 177,553 & 212,369 & 218,042 \\
\hline $\mathrm{Ni}, \mathrm{mg} / \mathrm{L}$ & $<0,0006$ & 0,0082 & $<0,0006$ & 0,0014 & 0,0012 \\
\hline $\mathrm{Rb}, \mathrm{mg} / \mathrm{L}$ & $0,3427 \pm 0,0903$ & 0,0103 & 0,1376 & 0,1217 & 0,1254 \\
\hline $\mathrm{Sb}, \mathrm{mg} / \mathrm{L}$ & $0,0342 \pm 0,0061$ & $<0,00013$ & 0,01254 & 0,00573 & 0,00573 \\
\hline $\mathrm{Si}, \mathrm{mg} / \mathrm{L}$ & $61,38 \pm 12,79$ & 31,17 & 37,90 & 29,81 & 30,40 \\
\hline $\mathrm{SiO}_{2}, \mathrm{mg} / \mathrm{L}$ & $131,32 \pm 27,37$ & 66,69 & 81,09 & 63,77 & 65,04 \\
\hline $\mathrm{Sr}, \mathrm{mg} / \mathrm{L}$ & $0,9115 \pm 0,1817$ & 1,0223 & 0,7426 & 0,9026 & 0,9340 \\
\hline $\mathrm{Ti}, \mathrm{mg} / \mathrm{L}$ & $0,0083 \pm 0,0014$ & 0,0034 & 0,0146 & 0,0050 & 0,0049 \\
\hline $\mathrm{U}, \mathrm{mg} / \mathrm{L}$ & $0,000417 \pm 0,000258$ & 0,000586 & 0,000111 & 0,000907 & 0,000862 \\
\hline $\mathrm{V}, \mathrm{mg} / \mathrm{L}$ & $0,0037 \pm 0,0003$ & 0,0036 & 0,0036 & 0,0023 & 0,0026 \\
\hline $\mathrm{W}, \mathrm{mg} / \mathrm{L}$ & $0,0061 \pm 0,0084$ & 0,0108 & $<0,0006$ & $<0,0006$ & $<0,0006$ \\
\hline $\mathrm{Yb}, \mathrm{mg} / \mathrm{L}$ & $<0,00006$ & 0,00012 & 0,00006 & $<0,00006$ & $<0,00006$ \\
\hline $\mathrm{Zn}, \mathrm{mg} / \mathrm{L}$ & $<0,0026$ & 0,0244 & 0,0216 & 0,0031 & $<0,0026$ \\
\hline
\end{tabular}

Los resultados obtenidos muestran que los elementos: $\mathrm{Ag}(<0,00001 \mathrm{mg} / \mathrm{L}), \mathrm{Bi}(<0,00003$ $\mathrm{mg} / \mathrm{L}), \mathrm{Cd}(<0,00003 \mathrm{mg} / \mathrm{L}), \mathrm{Hg}(<0,00009 \mathrm{mg} / \mathrm{L}), \mathrm{La}(<0,0015 \mathrm{mg} / \mathrm{L}), \mathrm{Lu}(<0,00006 \mathrm{mg} / \mathrm{L})$, $\mathrm{Nb}(<0,0015 \mathrm{mg} / \mathrm{L}), \mathrm{P}(<0,047 \mathrm{mg} / \mathrm{L}), \mathrm{Sn}(<0,00010 \mathrm{mg} / \mathrm{L}), \mathrm{Te}(<0,003 \mathrm{mg} / \mathrm{L})$ y $\mathrm{Tl}(<0,00006$ $\mathrm{mg} / \mathrm{L})$, en los 14 puntos de muestreo, se encontraron bajo el límite de cuantificación (LC). Los elementos $\mathrm{Pb}(0,0011 \mathrm{mg} / \mathrm{L}), \mathrm{Cr}(0,0069 \mathrm{mg} / \mathrm{L})$ y $\mathrm{Th}(0,03045 \mathrm{mg} / \mathrm{L})$ presentaron contenidos sobre el límite de cuantificación (LC), $\mathrm{Pb}(<0,0006 \mathrm{mg} / \mathrm{L}), \mathrm{Cr}(<0,0003 \mathrm{mg} / \mathrm{L})$ y Th $(<0,00019 \mathrm{mg} / \mathrm{L})$ en los puntos P9 $(\mathrm{Pb})$ y P5 (Cr y Th). Los elementos Se $(<0,0013$ $\mathrm{mg} / \mathrm{L}), \operatorname{Zr}(<0,00045 \mathrm{mg} / \mathrm{L})$ y $\mathrm{Ta}(<0,00021 \mathrm{mg} / \mathrm{L})$ presentaron concentraciones bajo el límite 
de cuantificación (LC) en la cuenca hidrográfica Sama. En la cuenca hidrográfica Locumba solamente los elementos Be $(0,00022 \mathrm{mg} / \mathrm{L})$ y Ta $(0,0052 \mathrm{mg} / \mathrm{L})$ en los puntos P1 y P5 presentaron valores sobre el límite de cuantificación (LC) Be $(<0,0006 \mathrm{mg} / \mathrm{L})$ y Ta $(<0,00021$ $\mathrm{mg} / \mathrm{L})$. Los límites de cuantificación (LC) para los otros elementos analizados fueron: As $(0,00010 \mathrm{mg} / \mathrm{L}), \mathrm{B}(0,006 \mathrm{mg} / \mathrm{L}), \mathrm{Ba}(0,0003 \mathrm{mg} / \mathrm{L}), \mathrm{Ca}(0,009 \mathrm{mg} / \mathrm{L}), \mathrm{Cs}(0,0003 \mathrm{mg} / \mathrm{L}), \mathrm{K}$ (0,13 mg/L), Li (0,0003 mg/L), Mg (0,003 mg/L), Mo (0,00006 mg/L), Na (0,019 mg/L), Rb (0,0009 mg/L), Si (0,13 mg/L), SiO2 (0,27 mg/L), Sr (0,0006 mg/L), Ti (0,0006 mg/L), U $(0,00001 \mathrm{mg} / \mathrm{L})$ y $\mathrm{V}(0,0003 \mathrm{mg} / \mathrm{L})$.

En las figuras 3 y 4 se presentan los resultados promedio de dos campañas de muestreo para el análisis de metales totales (mg/L M. Total), disueltos o solubles (mg/L M. Disuelto) y el porcentaje de metales insolubles (\% Insoluble) realizados en las muestras de aguas de los dos ríos salados; uno a finales de septiembre y el otro a inicios de diciembre. Los ríos Salado (P4 y P10) muestran claramente que los metales de valor añadido $\mathrm{Li}, \mathrm{Ge}, \mathrm{Rb}$ y $\mathrm{Cs}$ se encuentran en forma predominantemente soluble correspondiendo a su parte insoluble porcentajes de $17 \%, 10 \%, 2 \%$ y $2 \%$ respectivamente para $\mathrm{P} 4$ y porcentajes de $19 \%, 17 \%$, $5 \%$ y $5 \%$ respectivamente para $\mathrm{P} 10$. Estos resultados indican que un proceso único de microfiltración no sería suficiente para recuperarlos. Otros elementos como el Al, W, Fe, Ti, Co, Mo y Zn muestran, en los puntos P4 y P10, valores en su fracción insoluble en el rango de $98 \%$ a $37 \%$, siendo valores importantes para hacer posible su separación o recuperación mediante un proceso de microfiltración. En el punto P4 los elementos Al, W, Ti, Co, Zn, Mo y Sr mostraron valores entre $135 \%$ a $20 \%$ en el porcentaje del coeficiente de variabilidad de metales disueltos (\%CV M.D.) y en el punto P10 los elementos Ti, W, U, Mn, V y Sr mostraron valores de \%CV M.D. en el rango de $118 \%$ a $20 \%$; significando una variabilidad importante de estos elementos en los análisis realizados. En lo que respecta al As y B en los dos puntos $\mathrm{P} 4$ y P10 solamente es posible remover por un proceso de microfiltración un 14\% de As y 32\% de B. El comportamiento de los dos ríos salados (P4 y P10) muestran mayor estabilidad en el porcentaje del coeficiente de variación para metales totales (\%CV M.T.) que en (\%CV M.D.) 


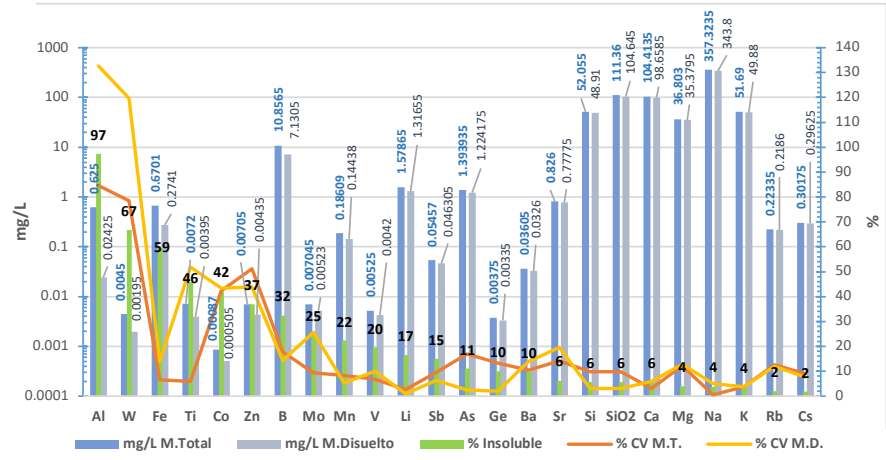

Figura 3. Concentraciones de metales totales ( $\mathrm{mg} / \mathrm{L} \mathrm{M}$. Total), disueltos ( $\mathrm{mg} / \mathrm{L} \mathrm{M}$. disuelto), porcentaje de metal Insoluble (\% Insoluble), porcentaje coeficiente de variación metales totales

(\%CV M.T.) y porcentaje coeficiente de variación metales disueltos (\%CV M.T.) en el río Salado-Locumba (P4).

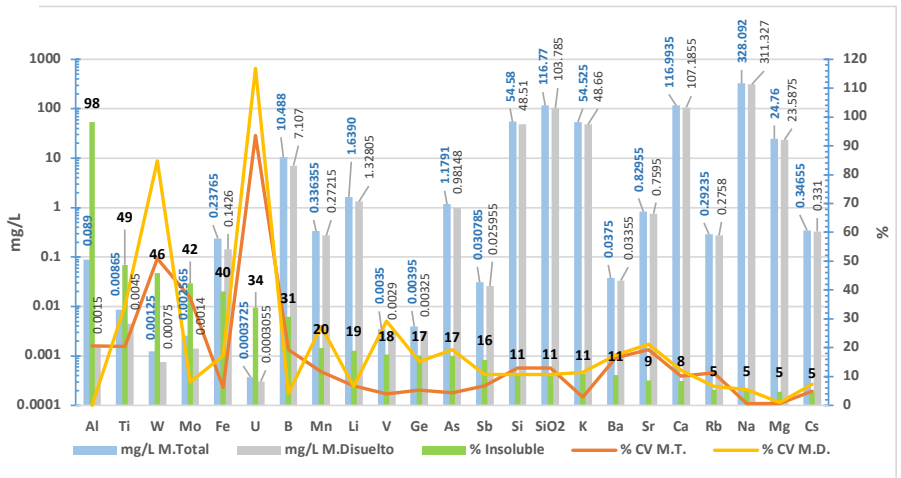

Figura 4. Concentraciones de metales totales ( $\mathrm{mg} / \mathrm{L} \mathrm{M}$. Total), disueltos ( $\mathrm{mg} / \mathrm{L} \mathrm{M}$. disuelto), porcentaje de metal insoluble (\% Insoluble), porcentaje del coeficiente de variación metales totales (\%CV M.T.) y porcentaje coeficiente de variación metales disueltos (\%CV M.T.) en el río Salado-Sama (P10).

\subsection{Correlación de los metales de valor añadido litio ( $\mathrm{Li})$, rubidio (Rb) y cesio (Cs) con los elementos Boro (B) y arsénico (As).}

En la figura 5 se puede observar varios elementos con valores atípicos ( $\mathrm{As}, \mathrm{Mg}, \mathrm{Rb}, \mathrm{Ba}, \mathrm{Co}$, $\mathrm{Cs}, \mathrm{Sb}, \mathrm{Ti}, \mathrm{U}$ y Sr). A los elementos cuyos datos no mostraron una distribución normal, se les aplicó la transformación de Box y Cox identificándolos anteponiendo la letra "T" (TAl, TBa, TCo, TCs, TFe, TSb, TTi, TU, TSr y TSi). Los elementos con distribución normal y valores atípicos fueron: $\mathrm{Mg}$, As y $\mathrm{Rb}$. 


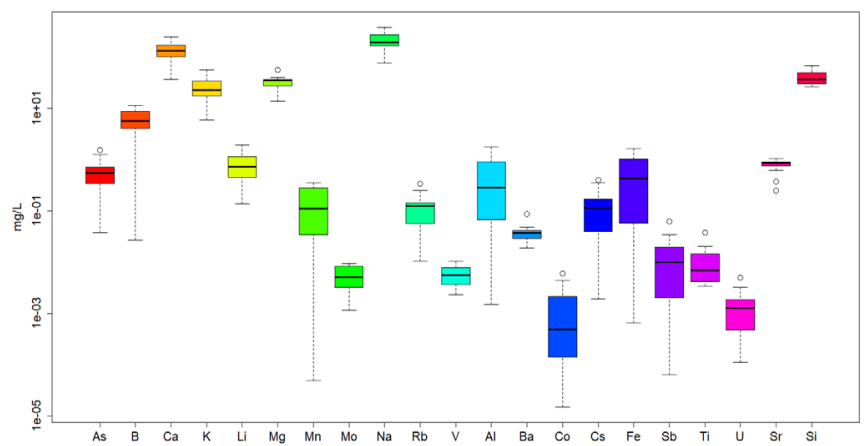

Figura 5. Gráfica de caja y bigotes para los elementos de los 14 puntos muestreados a lo largo de las cuencas hidrográficas Locumba y Sama.

La figura 6 muestra el grado de correlación entre los elementos mediante tonalidades de colores con valores en un rango de $-1 \mathrm{a}+1$. El nivel de significancia es mostrado en la figura 7 mediante el número de asteriscos $(*, * * \mathrm{y} * * *$ igual a $\mathrm{p}<0,05, \mathrm{p}<0,01 \mathrm{y} \mathrm{p}<0,001)$. Se puede observar que la correlación entre los elementos de valor añadido $\mathrm{Li}, \mathrm{Rb}, \mathrm{Cs}$ y los elementos As y B son estadísticamente significativos, positivos y altos ( $r>0,7$ y $p<0,01)$, el Ge no se consideró por mostrar valores bajo el límite de cuantificación (LC) en varios puntos del monitoreo. Debido a los valores atípicos mostrados por los elementos $\mathrm{Rb}$ y As, se realizó un análisis complementario aplicando una correlación robusta a los elementos de estudio.

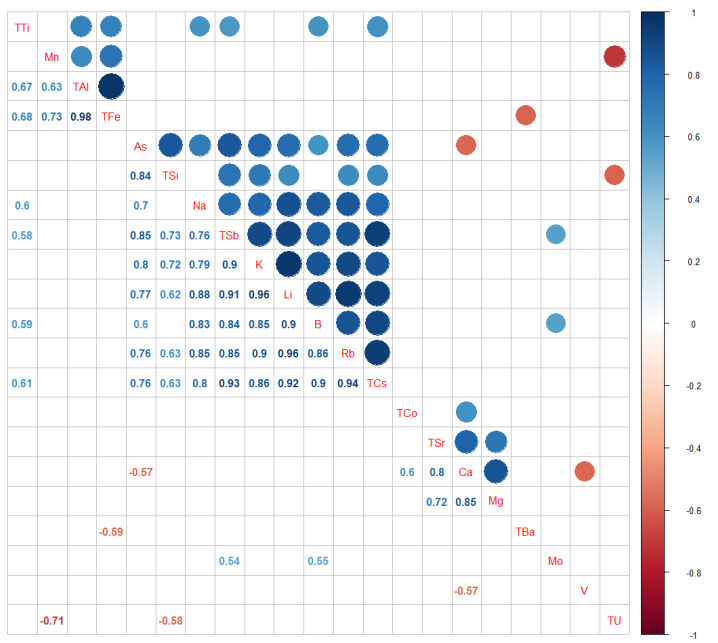

Figura 6. Correlación de Pearson de los elementos analizados en los 14 puntos de muestreo a lo largo de las cuencas hidrográficas Locumba y Sama (para $\mathrm{p}<0,05$ ). 


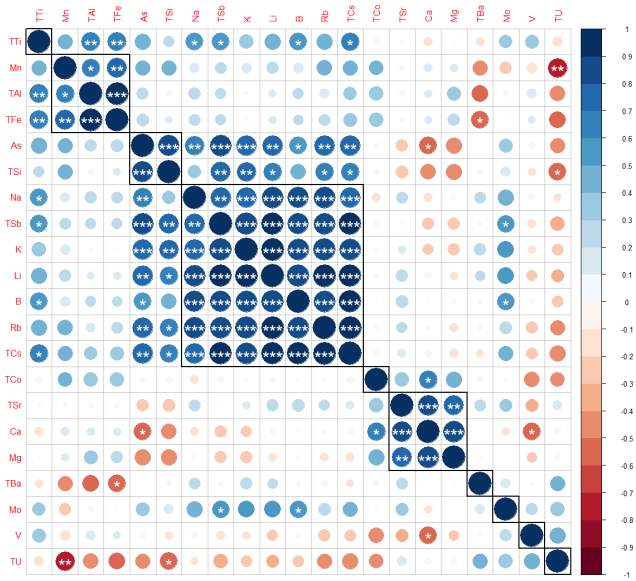

Figura 7. Nivel de significancia de los elementos analizados en los 14 puntos de muestreo a lo largo de las cuencas hidrográficas Locumba y Sama. Donde el nivel de significancia: *, ** y *** igual a $\mathrm{p}<0,05, \mathrm{p}<0,01$ y $\mathrm{p}<0,001$.

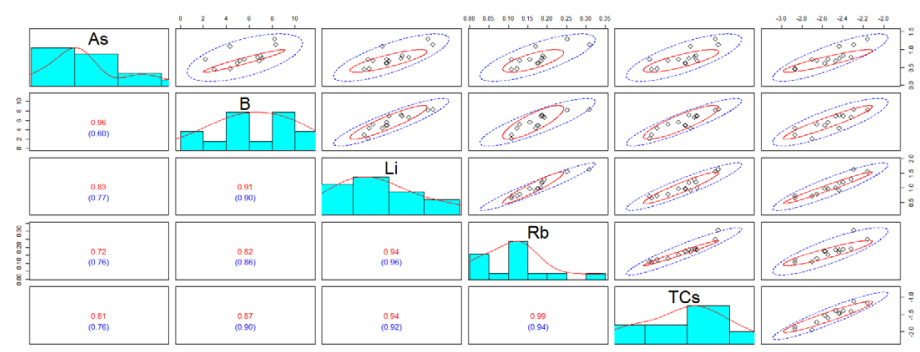

Figura 8. Correlación robusta de los elementos As, B, Li, Rb y Cs analizados en los 14 puntos de muestreo distribuidos a lo largo de las cuencas hidrográficas Locumba y Sama.

En la figura 8 se observan los valores de los coeficientes de correlación de Pearson (color azul), los coeficientes de correlación robustos (color rojo), distribución normal por elemento, elipses de color azul para el ámbito de la correlación de Pearson y color rojo para la correlación robusta. Para la correlación robusta, claramente, los valores atípicos se ubican fuera del área de las elipses rojas demostrando que existe un efecto de los mismos en los coeficientes de correlación de Pearson. La correlación robusta reduce el efecto de los valores atípicos mejorando, en general, el nivel de correlación entre los elementos seleccionados, siendo más evidente en los elementos Cs y As.

El análisis de componentes principales (PCA) se aplicó como complemento para la reducción del número de variables, análisis de correlación, determinación de diferencias y similitudes 
entre los sitios de muestreo. Siendo el número de elementos (variables) mayor que el número de puntos de muestreo (observaciones) y evidente la existencia de valores atípicos, se aplicó un análisis de componentes principales robusto (RobPCA), determinándose tres componentes principales identificados como PC1, PC2 y PC3, contribuyendo cada componente a la varianza en $68 \%, 29 \%$ y $3 \%$ respectivamente, se graficó las primeras dos componentes que juntas representan un total de $97 \%$ de la varianza (ver figura 9).

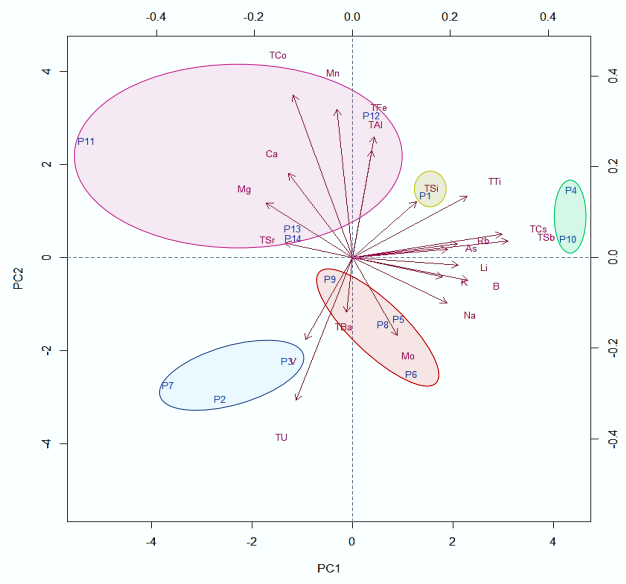

Figura 9. Biplot del análisis de componentes principales robusto (RobPCA) para los 14 puntos de muestreo a lo largo de las cuencas Locumba y Sama.

La figura 9 muestra los elementos representados por vectores. Ángulos cortos entre los vectores del grupo de elementos $\mathrm{Rb}, \mathrm{Cs}$ y As y un mayor ángulo de éste con los vectores de los elementos Li y B, significando en el primer caso un elevado nivel de correlación y en el segundo un nivel de correlación medio. En la misma figura se ha agrupado los 14 puntos de monitoreo según características semejantes entre sus elementos, siendo 5 agrupamientos (conglomerados) que están señalados por elipses de colores. Esta clasificación ha sido realizada mediante el análisis de agrupamiento jerárquico (HCA) con métrica Manhattan y método de Ward, esta configuración permite mayor tolerancia a datos atípicos. Los resultados se resumen en el dendograma de la figura 10; las agrupaciones (conglomerados) han sido identificados con números del 1 al 5, y conformadas por los puntos de muestreo: conglomerado $\mathrm{C}-1$ (P1), conglomerado C-2 (P2, P3 y P7), conglomerado C-3 (P4 y P10), conglomerado C-4 (P5, P6, P8 y P9) y conglomerado C-5 (P11, P12, P13 y P14). Observándose la similitud en las características químicas de los puntos de muestreo P4 y P10 conformando el conglomerado C-3. 


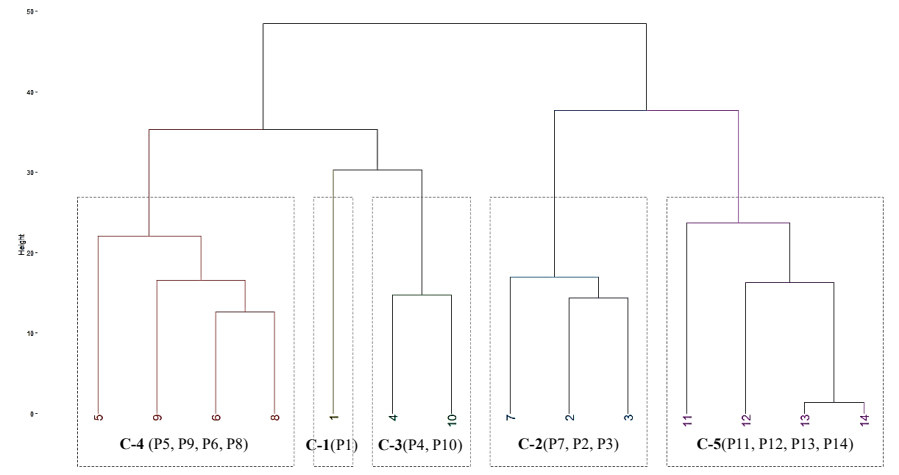

Figura 10. Dendograma de los 14 puntos de muestreo a lo largo de las cuencas hidrográficas Locumba y Sama. Identificando los puntos de muestreo (P1 al P14) con similares características que integran los 05 conglomerados (C-1 al C-5).

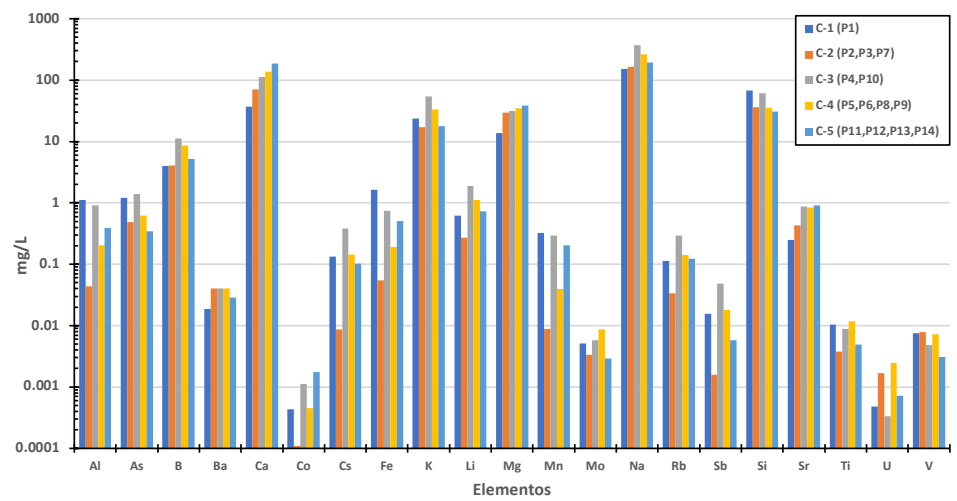

Figura 11. Distribución y mediana de la concentración en que se encuentran distribuidos en 05 conglomerados los elementos de los 14 puntos de muestreo a lo largo de las cuencas hidrográficas Locumba y Sama..

En la figura 11 es posible apreciar el valor de la mediana de los elementos químicos encontrados en los puntos de muestreo que conforman cada uno de los 05 conglomerados. Siendo posible identificar que los mayores valores para la mediana de la concentración de los elementos de valor añadido Li, Cs, y Rb se ubican en la barra de color gris de la figura 11, correspondiente al conglomerado C-3 integrado por los puntos de muestreo P4 y P10 (ríos Salado-Locumba y Salado-Sama). La figura 11 permite fácilmente determinar las características únicas de cada conglomerado como son los elementos que predominan en cada uno.

Al ser uno de los objetivos la identificación de los metales de valor añadido, se comparó con otros elementos traza en los 14 puntos de muestreo, observando que los metales $\mathrm{Sr}, \mathrm{Li}, \mathrm{Rb}$, Cs, Ti, V y U fueron los más abundantes, tal como lo representa la figura 12. 
En la figura 11 es posible apreciar el valor de la mediana de los elementos químicos encontrados en los puntos de muestreo que conforman cada uno de los 05 conglomerados. Siendo posible identificar que los mayores valores para la mediana de la concentración de los elementos de valor añadido $\mathrm{Li}, \mathrm{Cs}$, y Rb se ubican en la barra de color gris de la figura 11, correspondiente al conglomerado C-3 integrado por los puntos de muestreo P4 y P10 (ríos Salado-Locumba y Salado-Sama). La figura 11 permite fácilmente determinar las características únicas de cada conglomerado como son los elementos que predominan en cada uno.

Al ser uno de los objetivos la identificación de los metales de valor añadido, se comparó con otros elementos traza en los 14 puntos de muestreo, observando que los metales $\mathrm{Sr}, \mathrm{Li}, \mathrm{Rb}$, Cs, Ti, V y U fueron los más abundantes, tal como lo representa la figura 12 .

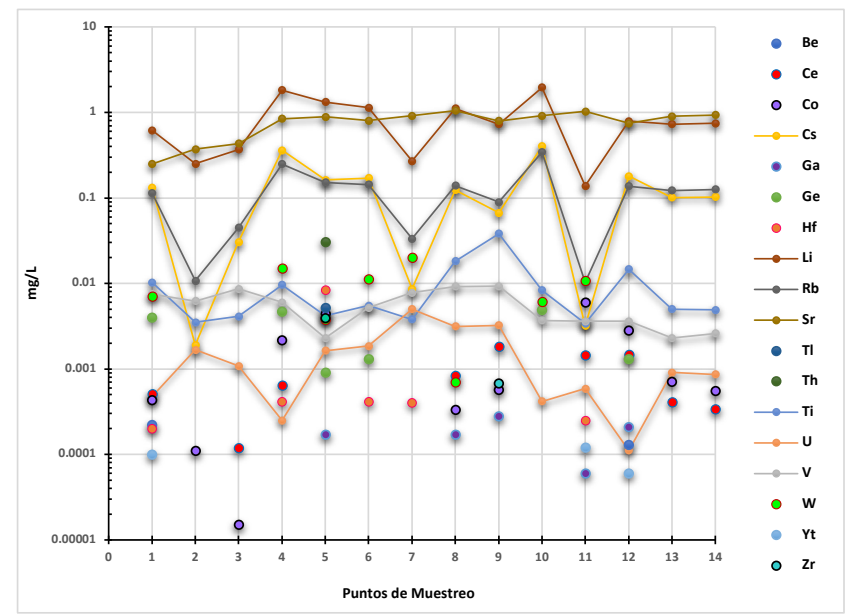

Figura 12. Identificación y concentración en que se encuentran todos los metales de valor añadido y otros elementos a nivel traza en los 14 puntos de muestreo ubicados en las cuencas hidrográfica Locumba y Sama.

En general no se ha encontrado un estudio que correlacione específicamente los elementos As y B con los metales de valor añadido Rb, Cs y Li. Como se mencionó estos elementos se encuentran normalmente en agua de origen geotermal que, al mezclarse con aguas superficiales, contribuyen a elevar la concentración de los mismos, como es el presente caso.

La calidad química de los 14 puntos de muestreo ubicados en diferentes fuentes de agua a lo largo de las cuencas hidrográficas Locumba y Sama, evidencian la presencia de estos elementos (figura 11 y 12). Las investigaciones realizadas ${ }^{(10)(11)}$ en agua subterránea, encontraron una buena correlación entre el As total y los elementos traza F, V, Mo, B y Si. Así como la existencia de autocorrelación entre los elementos B, As y Sb. En la misma línea, la 
investigación realizada ${ }^{(12)}$ reportó que, existe una buena autocorrelación entre los elementos As, Li, B y F presentes en agua geotermal. Es evidente que las investigaciones se plantean de acuerdo a un interés específico. Las investigaciones encontradas relacionadas con los elementos de estudio de la presente investigación, están orientadas al estudio hidrogeoquímico de aguas geotermales o subterráneas donde el objetivo es distinto al presente estudio; que no se centra en determinar el origen de estos elementos sino en identificarlos y cuantificarlos para su potencial extracción y comercialización, así como encontrar el grado de correlación de los mismos con dos elementos indicadores de calidad importantes como son el As y B encontrados en los 14 puntos de muestreo seleccionados y que superan en la mayoría de los casos el valor de $0,01 \mathrm{mg} / \mathrm{L}$ y $0,05 \mathrm{mg} / \mathrm{L}$ respectivamente, recomendado por la Organización Mundial de la Salud (OMS).

\subsection{Potenciales metales de valor añadido a extraer y comercializar}

Con la finalidad de poder analizar los valores obtenidos se realiza una comparación con valores referenciales de otros estudios de donde normalmente son extraídos para su comercialización. En la Tabla 3 se observa la comparación entre la concentración de algunos elementos en la salmuera proveniente de la desalinización a través de plantas de osmosis inversa (RO) que tratan el agua del mar Mediterráneo ${ }^{(13)}$ y los valores promedio encontrados en los ríos Salado (P4 y P10). Es evidente que las concentraciones de los metales Li, Rb, Cs y Ge en los ríos Salado-Locumba y Salado-Sama sin ningún tratamiento se encuentran en concentraciones superiores a los encontrados en la salmuera; mostrándose que se obtendría potencialmente concentraciones más elevadas de los elementos $\mathrm{Li}, \mathrm{Rb}, \mathrm{Cs}$ y Ge en la salmuera de rechazo, si se somete el agua de los ríos Salado-Locumba y Salado-Sama a un proceso de osmosis inversa u otro proceso de concentración.

Tabla 3. Promedio de concentración de algunos elementos presentes en la salmuera de rechazo proveniente de tratamiento de agua de mar por osmosis inversa y valores en el agua superficial de los ríos Salado-Locumba y Salado-Sama.

\begin{tabular}{|c|c|c|c|}
\hline \multirow[t]{2}{*}{ Metal } & \multirow[t]{2}{*}{$\begin{array}{c}\begin{array}{c}\text { Concentración en } \\
\text { salmuera de } \\
\text { desalación de agua de } \\
\text { mar }^{(13)}(\mathrm{mg} / \mathrm{L})\end{array} \\
\text { Mar mediterráneo } \\
\end{array}$} & \multicolumn{2}{|c|}{$\begin{array}{c}\text { Concentración mg/L en los ríos Salado- } \\
\text { Locumba y Salado-Sama (P4 y P10) de } \\
\text { las cuencas hidrográficas Locumba y } \\
\text { Sama }\end{array}$} \\
\hline & & P4 & P10 \\
\hline $\mathbf{L i}$ & 0,27 & $1,818 \pm 0,415$ & $1,9501 \pm 0,5445$ \\
\hline $\mathbf{R b}$ & 0,19 & $0,2484 \pm 0,0477$ & $0,3427 \pm 0,0903$ \\
\hline Cs & 0,0008 & $0,3606 \pm 0,1036$ & $0,4014 \pm 0,0958$ \\
\hline Ge & 0,00006 & $0,0047 \pm 0,0017$ & $0,0049 \pm 0,0016$ \\
\hline
\end{tabular}




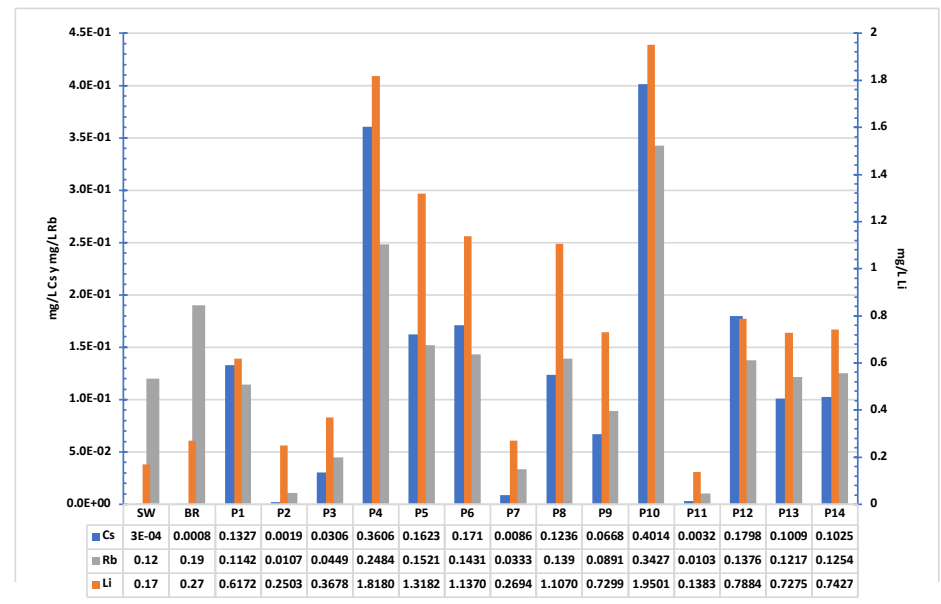

Figura 13. Concentración de los elementos $\mathrm{Cs}, \mathrm{Rb}$ y Li en los 14 puntos de muestreo, agua de mar $(\mathrm{SW})^{(16)}$ y en una salmuera $(\mathrm{BR})^{(13)}$ proveniente del agua de rechazo del tratamiento de osmosis inversa de agua de mar.

Los costos en el mercado internacional ${ }^{(14)}$ de los metales de valor añadido identificados con potencial proyección de ser extraídos o recuperados para su comercialización, serían los siguientes: rubidio $5980 \$ / \mathrm{kg}$ (como $\mathrm{RbCl}$ ), cesio $2072 \$ / \mathrm{kg}$ (como CsCl), germanio $920 \$ / \mathrm{kg}$ $\left(\mathrm{GeO}_{2}\right)$ y litio $13 \$ / \mathrm{kg}\left(\right.$ como $\left.\mathrm{Li}_{2} \mathrm{CO}_{3}\right)$. Algo que se advierte en los reportes internacionales ${ }^{(14)}$ de costos de estos elementos, es que si bien algunos de ellos tienen altos valores en el mercado internacional, el problema que puede darse es que existan años en los que el mercado se encuentre saturado del metal valioso o su consumo sea bajo. Por otra parte, el precio dependerá de la forma en que se desee el producto, del tipo de sal que tiene como elemento base al metal de valor añadido y la pureza. Según los valores en dólares por kilogramo antes mencionado, los metales de valor añadido con mayor costo en el mercado internacional en orden descendente son Rb, Cs, Ge y Li. Los metales de valor añadido identificados, en esta investigación, para su potencial extracción $\mathrm{Rb}, \mathrm{Cs}$ y $\mathrm{Li}$ se encuentran presente en los 14 puntos de muestreo. La figura 12 indica las concentraciones de estos metales en los 14 puntos de muestreo, los valores de estos elementos en un agua de mar (WR) ${ }^{(16)}$ y en salmuera (BR) resultado del proceso de osmosis inversa de agua de mar $^{(13)}$.

\section{CONCLUSIONES}

Los metales de valor añadido identificados y cuantificados en los ríos Salado-Locumba(P4) y Salado-Sama(P10) fueron: $\mathrm{Li}, \mathrm{Rb}, \mathrm{Cs}$ y Ge en concentraciones de 1,818 $\pm 0,415 \mathrm{mg} / \mathrm{L}$, $0,2484 \pm 0,0477 \mathrm{mg} / \mathrm{L}, 0,3606 \pm 0,1036 \mathrm{mg} / \mathrm{Ly} 0,0047 \pm 0,0017 \mathrm{mg} / \mathrm{L}$ para P4(Salado-Locumba) 
y $1,9501 \pm 0,5445 \mathrm{mg} / \mathrm{L}, 0,3427 \pm 0,0903 \mathrm{mg} / \mathrm{L}, 0,4014 \pm 0,0958 \mathrm{mg} / \mathrm{L}$ y $0,0049 \pm 0,0016$ para P10(Salado-Sama) respectivamente. Estos elementos por las concentraciones encontradas muestran potencial recuperación o extracción.

Se determinó que la correlación entre los elementos de valor añadido $\mathrm{Li}, \mathrm{Rb}$, Cs y los elementos As y B son estadísticamente significativos, positivos y altos ( $r>0,7$ y $p<0,01)$ en los 14 puntos de muestreo ubicados en diferentes fuentes de agua a lo largo de las dos cuencas hidrográficas. Lo que posibilita utilizar la misma, para calcular indirectamente los valores de la concentración de $\mathrm{Cs}$ y $\mathrm{Rb}$ a partir de las concentraciones de As, B y Li. Estos cálculos pueden ser aplicados para la construcción de valores históricos referenciales de $\mathrm{Rb}$ y Cs a partir de los valores históricos de los elementos As, B y Li determinados en los monitoreos de calidad de agua en las cuencas hidrográficas de Locumba y Sama desde el año 2011 al 2020. La ANA no muestra registro de los elementos Rb y Cs en su historial de calidad de agua, pero si valores para As, $\mathrm{B}$ y Li; haciendo posible construir y proyectar las concentraciones de $\mathrm{Rb}$ y Cs correspondientes.

\section{AGRADECIMIENTOS}

A la Universidad Nacional Jorge Basadre Grohman de Tacna por financiar a través de fondos de Canon, sobrecanon y regalías mineras esta investigación como parte del proyecto "Estudio de las especies de arsénico y evaluación de los metales de valor añadido en las cuencas de Locumba y Sama de la región Tacna”.

\section{REFERENCIAS BIBLIOGRÁFICAS}

1. Srivastava S, editor. Arsenic in Drinking Water and Food. Arsenic in Drinking Water and Food. Singapore: Springer; 2020. 486 p.

2. Bundschuh J, Litter MI, Parvez F, Román-Ross G, Nicolli HB, Jean JS, et al. One century of arsenic exposure in Latin America: A review of history and occurrence from 14 countries. Sci Total Environ. 2012;429:2-35.

3. González Lope S. Diseño de una planta piloto para la evaluación de procesos de extracción de metales de valor añadido en agua de mar [Internet]. Barcelona: Universidad Politecnica de Cataluña; 2010. [Citado 15 jun 2021]. Disponible en: http://upcommons. upc.edu/handle/2099.1/10043?locale-attribute=es

4. ANA (Autoridad Nacional del Agua Perú). Plan de gestión de los recursos hídricos de la cuenca Caplina - Locumba [Internet]. 2015. 262 p. [Citado 12 jul 2021]. Disponible en: http://repositorio.ana.gob.pe/handle/ANA/88

5. Luque G. Zonas criticas por peligros geológicos en la región Tacna [Internet]. Instituto Geológico, Minero y Metalúrgico - INGEMMET. 2016. [Citado 22 jun 2021]. Disponible en: https://hdl.handle.net/20.500.12544/2028

6. Consorcio Río Locumba. Estudio de los recursos hídricos superficiales y subterráneos e 
infraestructura hidráulica para el plan de aprovechamiento en la cuenca del río Locumba, en la región de Tacna: [Internet]. 2017; 09 [Citado 25 jun 2021]. Disponible en: http:// repositorio.ana.gob.pe/handle/ANA/3644

7. ANA. Protocolo nacional para el monitoreo de la calidad de los recursos hídricos superficiales [Internet]. Autoridad Nacional del Agua. 2016. p. 85. [Citado 8 jun 2021]. Disponible en: http://repositorio.ana.gob.pe/handle/20.500.12543/209

8. Hatipoğlu Temizel E, Gültekin F, Firat Ersoy A. Major, trace, and rare earth element geochemistry of the Ayder and İkizdere (Rize, NE Turkey) geothermal waters: Constraints for water-rock interactions. Geothermics. 2020;86:101810. doi:10.1016/j. geothermics.2020.101810

9. Kaasalainen H, Stefánsson A. The chemistry of trace elements in surface geothermal waters and steam, Iceland. Chem Geol. 2012;330-331:60-85.

10. Bhattacharya P, Claesson M, Bundschuh J, Sracek O, Fagerberg J, Jacks G, et al. Distribution and mobility of arsenic in the Río Dulce alluvial aquifers in Santiago del Estero Province, Argentina. Sci Total Environ. 2006;358(1-3):97-120.

11. Quino-Lima I, Ramos-Ramos O, Ormachea-Muñoz M, Quintanilla-Aguirre J, Duwig C, Maity JP, et al. Spatial dependency of arsenic, antimony, boron and other trace elements in the shallow groundwater systems of the Lower Katari Basin, Bolivian Altiplano. Sci Total Environ. 2020; 719:137505. doi:10.1016/j.scitotenv.2020.137505

12. Yuan J, Guo Q, Wang Y. Geochemical behaviors of boron and its isotopes in aqueous environment of the Yangbajing and Yangyi geothermal fields, Tibet, China. J Geochemical Explor. 2014;140:11-22.

13. Ortiz-Albo P, Torres-Ortega S, González Prieto M, Urtiaga A, Ibañez R. TechnoEconomic Feasibility Analysis for Minor Elements Valorization from Desalination Concentrates. Sep Purif Rev. 2019;48(3):220-241.

14. U.S Geological Survey. Mineral Commodity Summaries 2020. Reston, Virginia: Department of the Interior, U.S. Geological Survey (USGS); 2020.

15. TradeTech (Uranium prices \& analysis since 1968) [Internet]. [citado 16 jun 2021]. Disponible en: https://www.uranium.info/nuexco_exchange_value.php

16. Herbelio P. Especiación de metales pesados mediante la formación de quelatos Distribución de las especies inorganicas de As y $\mathrm{Cr}$ en la Ría de Arousa [Internet]. Santiago de Compostela; 2007. [citado 18 jun 2021]. Disponible en: https://dialnet. unirioja.es/servlet/tesis?codigo $=108686$ 